\title{
A FUNDAMENTAL PROBLEM OF NAVIGATION IN FREE SPACE*
}

\author{
BY \\ GEORGE M. EWING \\ University of Oklahoma Research Institute and U. S. Army Artillery and Missile School
}

1. Introduction. We are concerned with the problem, termed fundamental by D. F. Lawden [6, p. 171], of transferring a rocket-driven vehicle from an initial position and velocity to a terminal position and velocity in such a way as to minimize the mass ratio.

Mathematical models for this and other questions considered in recent publications (see Ref. [4] through [9] for examples) suggest problems of Bolza in the calculus variations but often are not covered by the formulation of Bliss [1, p. 189] as a result of the essential nonincreasing character of mass.

Treatments fall into: Type 1, in which mass $M$ is permitted to have discontinuities (as in $[4,5,6]$ ) and Type 2, in which a bound is placed on the rate of mass flow (as in $[7,8,9])$. Type 2 simulates an obvious limitation of rocket motors. Type 1 problems though less realistic in this respect are of interest per se, sometimes appear easier to attack, and furnish bounds on the performance provided by solutions of corresponding Type 2 problems. Type 2 problems have been treated (see [9, p. 9, items (2), (3)]) with the aid of side equalities equivalent to the restriction that mass $M$ be monotone, a device introduced by F. A. Valentine ([10, p. 4]) under more classical hypotheses. An alternative technique $([8$, p. 582]) termed parametric representation is due to Miele. Such approaches bring Type 2 problems closer to the classical fold. In contrast it appears that definitive treatments of Type 1 problems require new lines of attack.

The theory of optimal burning and steering programs is presently in an incomplete state. Many writers consider only the formal manipulation of first order conditions. There is no explicit statement of problems; hence the reader must decide from the context which functions are admissible and which steps are legitimate. Questions of existence and sufficiency are largely unmentioned. Miele ([7, pp. 105-109] and [8]) gives sufficiency arguments for certain problems.

In the formulation of Type 1 problems it seems desirable to admit general nonincreasing mass functions $M$. Neither $M$ nor the velocity is then necessarily $A C$ (absolutely continuous) in the time $t$. Conclusions based upon differential equations or otherwise involving the integration of $M^{\prime}$ or of accelerations can be correct only by coincidence.

The present paper establishes the existence of the absolute minimum for a Type 1 planar version of the problem mentioned at the beginning and shows that at least one optimal program is determined by certain solutions of an elementary system of equations, (3.2). The problem is partially covered by [4] and by the heuristic discussion of $[6$, pp. 171-175], subject to the objection of the preceding paragraph.

2. Formulation of the problem. Let $T$ denote the time required for a particle to go from point $(0,0)$ and velocity $(u, v)$ to position $(a, b)$ and velocity $(U, V)$. Time $T$ is preassigned except for Sec. 8 . We understand $T$ to be positive with the single exception that the trivial case $T=0$ is included under Theorem 5.4 as type (v).

* Received July 21, 1959; revised manuscript received November 12, 1959. 
An admissible burning program is any real function $M$ of time $t$ defined and nonincreasing for all real $t$ and having respective constant values $M(0-), M(T+)$ for $t<0,>T$.

An admissible steering program is any real function $\theta$ of $t$ defined and continuous for all real $t$. Continuity appears to be acceptable in the construction of a model and suffices for the needs of Sec. 6. This restriction could likely be relaxed with sufficient effort.

Let $\mu$ denote the measure generated by the non-decreasing function $-\ln M$ of an admissible $M$.

An admissible trajectory is any ordered pair of real functions $x, y$ of $t$ defined by an admissible $M$ and $\theta$ and the system of integral equations

$$
\begin{aligned}
& u(t)=c \int_{0}^{t} \cos \theta d \mu+u, \\
& v(t)=c \int_{0}^{t} \sin \theta d \mu+v, \\
& x(t)=\int_{0}^{t} u(\tau) d \tau, \quad y(t)=\int_{0}^{t} v(\tau) d \tau .
\end{aligned}
$$

If $M$ should have a continuous derivative $M^{\prime}$ then $d \mu$ could be replaced by $-M^{\prime} d \tau / M$ in (2.1). Familiar trajectory equations ([6, p. 172]) would follow by differentiation.

Constant $c$ is to be interpreted as magnitude of the exit velocity. Functions $u(t)$, $v(t)$ introduced is (2.1) are discontinuous when and only when $M$ is discontinuous. Clearly $u(0-)=u, v(0-)=v$. Moreover $|u(t)|,|v(t)|$ are bounded by $c \ln (M(0-) / M(T+))$; hence $x(t), y(t)$ are Lipschitzian and therefore $A C$ on any time-interval. We then know that $x$ and $y$ are differentiable a.e. (almost everywhere) and that $x^{\prime}(t)=u(t), y^{\prime}(t)=$ $v(t)$ a.e. on $[0, T]$ or any other interval.

If $M, \theta$ and the pair $x, y$ are all admissible the ordered quadruple $(M, \theta, x, y)$ will be termed admissible.

Problem. To minimize $M(0-) / M(T+)$ on the class of all admissible quadruples satisfying the fixed terminal conditions

$$
u(T)=U, \quad v(T)=V, \quad x(T)=a, \quad y(T)=b .
$$

Both the existence and the nature of a minimizing quadruple will be established via an auxiliary problem.

3. Formulation of the auxiliary problem. Let $n \geq 2$ be a fixed positive integer and consider the new problem obtained from that of Sec. 2 by restricting $M$ to be a step-function with at most $(n+1)$ steps at $t_{0}=0, t_{n}=T$, and at times $t_{1}, t_{2}, \cdots, t_{n-1}$, which may be anywhere on the closed interval $[0, T]$ and are not necessarily in the order indicated by subscripts. Let $r_{i}$ denote the mass-ratio at $t_{i}$, viz.

$$
r_{i}=M\left(t_{i}-\right) / M\left(t_{i}+\right), \quad i=0, \cdots, n .
$$

Integrals (2.1) are now expressible as sums on $i$ from 0 to $n$ and the auxiliary problem can be rephrased as the following ordinary minimum problem.

Auxiltary Problem. To minimize

$$
\sum \ln r_{i}=\ln (M(0-) / M(T+))
$$


subject to the constraint

$$
\left.\begin{array}{l}
\sum\left(T-t_{i}\right)\left(\cos \theta_{i}\right) \ln r_{i}=(a-u T) / c, \\
\sum\left(T-t_{i}\right)\left(\sin \theta_{i}\right) \ln r_{i}=(b-v T) / c, \\
\sum\left(\cos \theta_{i}\right) \ln r_{i}=(U-u) / c, \\
\sum\left(\sin \theta_{i}\right) \ln r_{i}=(V-v) / c,
\end{array}\right]
$$

and to the further restrictions

$$
\begin{gathered}
r_{i} \geq 1, \quad i=0,1, \cdots, n, \\
t_{0}=0, \quad t_{n}=T \quad \text { and } \quad 0 \leq t_{i} \leq T, \quad i=1, \cdots,(n-1) .
\end{gathered}
$$

Clearly the infimum of $M(0-) / M(T+)$ does not excede the particular value $K$ obtained by setting $r_{i}=1$ and assigning arbitrary values to $\theta_{i}, i=1, \cdots,(n-1)$, and completing the elementary solution of the four equations (3.2) for $r_{0}, r_{n}, \theta_{0}, \theta_{n}$. Neither the existence nor nature of solutions of the auxiliary problem is affected by adjoining the restriction

$$
r_{i} \leq K, \quad i=0, \cdots, n .
$$

Since $\theta_{i}$ enters only through the periodic functions sine and cosine we are similarly free to add the restriction

$$
0 \leq \theta_{i} \leq 2 \pi, \quad i=0, \cdots, n .
$$

Side conditions (3.2) through (3.6) define a bounded closed non-empty subset $G$ of $(3 n+1)$ - space; hence there exists a minimizing point

$$
\left(r_{0}, r_{1}, \cdots, r_{n}, \theta_{0}, \theta_{1}, \cdots, \theta_{n}, t_{1}, \cdots, t_{n-1}\right),
$$

in $G$ for the continuous function $\Sigma \ln r_{i}$.

Successive reductions of the minimizing set of values (3.7) are effected as follows.

If $t_{i}=0$ drop the $j$ th term in each equation (3.1), (3.2) at the same time replacing $\ln r_{0}$ in (3.1) by $\ln r_{0} r_{i}$ and adding $j$ th terms to 0 th terms vectorially in (3.2). Side conditions (3.2), (3.3), (3.4) continue to hold but the number of summands in (3.1), (3.2) is reduced by unity. Similar remarks apply if $t_{i}=T$ or more generally if $t_{j}=t_{k}$.

If $r_{i}=1, j \neq 0$ or $n$ the $j$ th terms in (3.1), (3.2) are understood to be deleted. It is convenient to retain first and last terms in these sums as objects of discussion even when such terms equal zero.

Finally if $\theta_{i}=\theta_{k} \bmod 2 \pi, j \neq k$, replace the pairs of terms in (3.1), (3.2) by a single term in each case using the solution $t, r$ of the system

$$
\begin{gathered}
(T-t) \ln r=\left(T-t_{j}\right) \ln r_{i}+\left(T-t_{k}\right) \ln r_{k}, \\
\ln r=\ln r_{j}+\ln r_{k} .
\end{gathered}
$$

One verifies that

$$
\left(t-t_{j}\right):\left(t_{k}-t\right)=\ln r_{k}: \ln r_{j}=\left(t_{i}-t\right):\left(t-t_{k}\right) ;
$$

hence that $t$ is on the interval $[0, T]$. 
Reductions of the several types can be applied and remaining values (3.7) relabeled so as to yield a reduced set

$$
\left(r_{0}, r_{1}, \cdots, r_{m}, \theta_{0}, \theta_{1}, \cdots, \theta_{m}, t_{1}, \cdots, t_{m-1}\right), \quad m \leq n,
$$

satisfying the restrictions

$$
\begin{aligned}
& r_{0} \geq 1, \quad r_{m} \geq 1 ; \quad r_{i}>1, \quad i=1, \cdots,(m-1), \\
& \theta_{i} \neq \theta_{k} \quad \bmod 2 \pi, \quad j \neq k, \\
& t_{0}=0, \quad t_{m}=T \\
& 0<t_{i}<T, \quad i=1, \cdots,(m-1), \\
& t_{i} \neq t_{k}, \quad j \neq k .
\end{aligned}
$$

By the reduced auxiliary problem or reduced problem for short we mean the problem of minimizing (3.1) subject to side conditions (3.2), (3.9), (3.10), (3.11). The range of summation will now be denoted by $m$ as a reminder of the reduction process. Since the auxiliary problem has a solution the reduced problem necessarily has a solution. Moreover a solution of the reduced problem is a solution of the original auxiliary problem.

4. Application of Lagrange multipliers. Consider the problem $f(x)=$ minimum subject to constraints $\varphi_{1}(x)=0, \cdots, \varphi_{\alpha}(x)=0$, in which $x$ denotes $\left(x_{1}, \cdots, x_{\beta}\right)$ and $\alpha<\beta$. The usual multiplier process yields a necessary condition on a minimizing point $X$ provided that (see for example [2, pp. 544-547]) $X$ is an interior point of the set on which a minimum is sought and provided that the $\alpha$ by $\beta$ matrix of derivatives of $\varphi_{1}, \cdots, \varphi_{\alpha}$ with respect to $x_{1}, \cdots, x_{\beta}$ is of rank $\alpha$.

In the proofs of Sec. 5 the interior nature of the minimizing point follows from selected strict inequalities (3.9), (3.11). One can verify that the matrix has the required rank in each case. Further mention of this condition omitted.

Let $R, A, B, C, D$ denote respective left members of (3.1), (3.2) for the reduced problem, let $\lambda_{1}, \cdots, \lambda_{4}$ be multipliers, and let $F \equiv R+\lambda_{1} A+\lambda_{2} B+\lambda_{3} C+\lambda_{4} D$. We record for reference that

$$
\begin{gathered}
\partial F / \partial r_{i}=\frac{1}{r_{i}}\left[1+\lambda_{1}\left(T-t_{i}\right) \cos \theta_{i}+\lambda_{2}\left(T-t_{i}\right) \sin \theta_{i}+\lambda_{3} \cos \theta_{i}+\lambda_{4} \sin \theta_{i}\right] \\
\partial F / \partial \theta_{i}=\ln r_{i}\left[-\lambda_{1}\left(T-t_{i}\right) \sin \theta_{i}+\lambda_{2}\left(T-t_{i}\right) \cos \theta_{i}-\lambda_{3} \sin \theta_{i}+\lambda_{4} \cos \theta_{i}\right] \\
\partial F / \partial t_{i}=-\ln r_{i}\left[\lambda_{1} \cos \theta_{i}+\lambda_{2} \sin \theta_{i}\right] .
\end{gathered}
$$

We shall use certain of the equations

$$
\begin{array}{ll}
\partial F / \partial r_{i}=0, & i=0, \cdots, m, \\
\partial F / \partial \theta_{i}=0, & i=0, \cdots, m, \\
\partial F / \partial t_{i}=0, & i=1, \cdots,(m-1) .
\end{array}
$$

5. Properties of a solution of the reduced auxiliary problem.

Let

$$
\left(r_{0}, \cdots, r_{m}, \theta_{0}, \cdots, \theta_{m}, t_{1}, \cdots, t_{m-1}\right),
$$

denote a solution of the reduced problem. 
Theorem 5.1. A solution (5.1) can include at most one mass ratio $r_{i}$ greater than unity whose subscript differs from 0 or $m$.

Proof. Suppose that $r_{i}>1, r_{k}>1, j \neq l$, and that neither $j$ nor $k$ is 0 or $m$. Consider the minimum of (3.1) in six variables $r_{i}, \theta_{i}, t_{i}, i=j, k$ subject to four constraints (3.2) with other variables fixed at values (5.1).

From (4.3) with $i=j, k$

$$
\begin{aligned}
& \lambda_{1} \cos \theta_{i}+\lambda_{2} \sin \theta_{i}=0, \\
& \lambda_{1} \cos \theta_{k}+\lambda_{2} \sin \theta_{k}=0 .
\end{aligned}
$$

It follows that either $\lambda_{1}=\lambda_{2}=0$ or in view of (3.10) that $\theta_{i}, \theta_{k}$ differ by an odd multiple of $\pi$.

Under the first alternative we see from (4.1), (4.2) with $i=j$ that $\lambda_{3}=-\cos \theta_{i}$ and $\lambda_{4}=-\sin \theta_{i}$. Similarly with $i=k, \lambda_{3}=-\cos \theta_{k}$ and $\lambda_{4}=-\sin \theta_{k}$. Such results contradict (3.10); hence cannot occur.

Under the second alternative we have from (4.3) and (4.1) for $i=j$ that $\lambda_{3} \cos$ $\theta_{i}+\lambda_{4} \sin \theta_{j}=-1$ and for $i=k$ the contradiction that $\lambda_{3} \cos \theta_{k}+\lambda_{4} \sin \theta_{k}=-\lambda_{3} \cos$ $\theta_{i}-\lambda_{4} \sin \theta_{i}=-1$.

TheOREM 5.2. If $r_{0}>1$ in a solution (5.1) then $r_{i}=1, i=1, \cdots,(m-1)$.

Proof. Suppose that $r_{0}>1$ and that $r_{j}>1$ for some fixed $j$ between 0 and $(m-1)$ inclusive. Consider the minimum problem in five variables $r_{0}, r_{i}, \theta_{0}, \theta_{i}, t_{i}$ with all other variables fixed at values (5.1). From the five equations consisting of (4.1), (4.2) for $i=0, j$ and (4.3) for $i=j$ we obtain relations

$$
\begin{gathered}
\lambda_{1} \cos \theta_{i}+\lambda_{2} \sin \theta_{i}=0, \\
\lambda_{3} \cos \theta_{i}+\lambda_{4} \sin \theta_{i}=-1, \\
\left(T-t_{0}\right)\left(\lambda_{1} \cos \theta_{0}+\lambda_{2} \sin \theta_{0}\right)+\lambda_{3} \cos \theta_{0}+\lambda_{4} \sin \theta_{0}=-1, \\
\left(T-t_{0}\right)\left(-\lambda_{1} \sin \theta_{0}+\lambda_{2} \cos \theta_{0}\right)-\lambda_{3} \sin \theta_{0}+\lambda_{4} \cos \theta_{0}=0, \\
\left(T-t_{j}\right)\left(-\lambda_{1} \sin \theta_{j}+\lambda_{2} \cos \theta_{i}\right)-\lambda_{3} \sin \theta_{i}+\lambda_{4} \cos \theta_{i}=0 .
\end{gathered}
$$

Solving (5.2) and (5.5) for $\lambda_{1}, \lambda_{2}$ we find that

$$
\left.\begin{array}{l}
\lambda_{1}=-\left(\sin \theta_{i}\right)\left(\lambda_{3} \sin \theta_{0}-\lambda_{4} \cos \theta_{0}\right) /\left(T-t_{0}\right) \cos \left(\theta_{i}-\theta_{0}\right), \\
\lambda_{2}=\left(\cos \theta_{i}\right)\left(\lambda_{3} \sin \theta_{0}-\lambda_{4} \cos \theta_{0}\right) /\left(T-t_{0}\right) \cos \left(\theta_{i}-\theta_{0}\right) .
\end{array}\right)
$$

If $\cos \left(\theta_{i}-\theta_{0}\right)$ were zero then $\lambda_{3} \sin \theta_{0}-\lambda_{4} \cos \theta_{0}=0$, which contradicts the result obtained from (5.3) by replacing $\cos \theta_{i}, \sin \theta_{i}$ with $-\sin \theta_{0}, \cos \theta_{0}$. Thus denominators in (5.7) cannot vanish.

From (5.6), (5.7)

$$
\begin{aligned}
& \lambda_{3}\left[\sin \theta_{i}-\left(T-t_{i}\right)\left(\sin \theta_{0}\right) /\left(T-t_{0}\right) \cos \left(\theta_{i}-\theta_{0}\right)\right] \\
& +\lambda_{4}\left[-\cos \theta_{i}+\left(T-t_{j}\right)\left(\cos \theta_{0}\right) /\left(T-t_{0}\right) \cos \left(\theta_{i}-\theta_{0}\right)\right]=0,
\end{aligned}
$$

while from (5.8), (5.3) with the fact that $t_{0}=0$,

$$
\left.\begin{array}{l}
\lambda_{3}=\left(T / t_{j}\right)\left[-\cos \theta_{j}+\left(T-t_{j}\right)\left(\cos \theta_{0}\right) / T \cos \left(\theta_{j}-\theta_{0}\right)\right], \\
\lambda_{4}=\left(T / t_{i}\right)\left[-\sin \theta_{i}+\left(T-t_{i}\right)\left(\sin \theta_{0}\right) / T \cos \left(\theta_{j}-\theta_{0}\right)\right] .
\end{array}\right)
$$


Substitute (5.9) into (5.7) and find that

$$
\lambda_{1}=-t_{i}^{-1} \sin \theta_{i} \tan \left(\theta_{i}-\theta_{0}\right), \quad \lambda_{2}=t_{i}^{-1} \cos \theta_{i} \tan \left(\theta_{i}-\theta_{0}\right) .
$$

Finally substitute (5.10) and (5.9) into (5.4), which reduces to $\cos \left(\theta_{j}-\theta_{0}\right)=1$, in contradiction with (3.10).

TheOREM 5.3. If $r_{m}>1$ in a solution (5.1) then $r_{i}=1, i=1, \cdots,(m-1)$.

Proof. The proof follows that of Theorem 5.2 as far as (5.6) with subscript 0 replaced by $m$. We now understand this change to have been made in (5.2), $\cdots,(5.6)$.

The proof now diverges as a result of the fact that $\left(T-t_{m}\right)=0$. From (5.4), (5.5) accordingly reduced $\lambda_{3}=-\cos \theta_{m}, \lambda_{4}=-\sin \theta_{m}$, which with (5.3) again contradicts (3.10).

The following theorem summarizes results of Sec. 3, 5. For completeness the trivial case in which $T=0$ and $m=1$, thus far excluded from our formulation, is included as (v).

THEOREM 5.4. The auxiliary problem necessarily has a solution of one of the following types

(i) $r_{0}>1, r_{m}>1$, each other ratio $=1$,

(ii) $r_{0}>1$, each other ratio $=1$,

(iii) $r_{m}>1$, each other ratio $=1$,

(iv) $r_{i}>1$ for one $j \neq 0, m$ and each other ratio $=1$,

(v) $m=1, r_{0} \geq 1$ and each other ratio $=1$.

THEOREM 5.5. If the auxiliary problem has a type (iv) minimum it also has a type (i) minimum.

Proof. Let $h, H$ respectively denote the expressions $a v-b u, a V-b U$ and let $R$ denote the positive square root of $(U-u)^{2}+(V-v)^{2}$. If a type (iv) solution exists elementary relations among distance, speed, and time imply that

Moreover

$$
T=(h-H) /(v U-u V) \text {. }
$$

$$
c\left(\min \sum \ln r_{i}\right)=R .
$$

If we now solve (3.2) with $m=2$ and $r_{1}=1$, taking the preassigned $T$ to be (5.11) we find that

$$
c \sum \ln r_{i}=R\left\{\left[h^{2} /(h-H)^{2}\right]^{1 / 2}+\left[H^{2} /(h-H)^{2}\right]^{1 / 2}\right\} .
$$

The stated conclusion follows from (5.12) and the fact that the above expression in braces cannot exceed unity.

6. Solution of the original problem. Let $(M, \theta, x, y)$ be any admissible quadruple satisfying terminal conditions (2.2). It is convenient to the application below of a convergence theorem for Stjeltjes integrals to consider a sequence $M_{v}$ of step functions converging to $M I$ or the interval $[-1, T+1]$ except possibly at discontinuities of $M$. (Any other closed interval to which $[0, T]$ is interior would do.) Such a sequence $M$, can be obtained using a suitable nested sequence $P_{\nu}$ of partitions of $[0, T]$. Define $M_{\nu}(t)$ for $-1 \leq t \leq 0$ as $M(0-)$, for $T<t \leq T+1$ as $M(T+)$ and for each of the disjoint half-open subintervals whose union is the half-open interval $[0, T]$ as the mean of $M$ over that subinterval. Let $\theta_{\nu}$ be any sequence of continuous functions converging uniformly on $[-1, T+1]$ to $\theta$; in particular take $\theta_{\nu}=\theta, \nu=1,2, \cdots$.

A well-known theorem $([3$, p. 283 , Theorem 23$])$ insures that the sequences 


$$
\begin{aligned}
& u_{\nu}(t)=c \int_{0}^{t} \cos \theta_{\nu} d \mu_{\nu}+u, \\
& v_{\nu}(t)=c \int_{0}^{t} \sin \theta_{\nu} d \mu_{\nu}+v,
\end{aligned}
$$

converge for $t \varepsilon[-1, T+1]$ except for $t$ a point of discontinuity of $M$. (See Hypothesis $B_{1}$, [3], p. 281). Moreover limits of right members of (6.1) are right members of the first two equations (2.1) written for the $M, \theta$ given above except for the countable set of discontinuities of $M$.

The ordinary bounded convergence theorem then insures that

$$
x_{\nu}(t)=\int_{0}^{t} u_{\nu}(\tau) d \tau, \quad y_{\nu}(t)=\int_{0}^{t} v_{\nu}(\tau) d \tau,
$$

converge to the admissible trajectory $x, y$ included in the admissible quadruple with which we started.

The position $\left(a_{v}, b_{v}\right)$ given by (6.2) and velocity $\left(U_{v}, V_{v}\right)$ given by $(6.1)$ for $t=T$ may differ from values $(2.2)$ but $u_{\nu}(T+), v_{\nu}(T+), x_{\nu}(T), y_{\nu}(T)$ necessarily converge to respective values (2.2).

By Theorem 5.4 there is a step function $M^{*}$ with at most two steps and a continuous function $\theta_{\nu}^{*}$ whose values at the discontinuities of $M^{*}$, together with $M^{*}$ define a solution to the auxiliary problem with terminal position $\left(a_{\nu}, b_{v}\right)$ and terminal velocity $\left(U_{\nu}, V_{\nu}\right)$ at $T$. Hence

$$
\ln \left[M_{\nu}^{*}(0-) / M_{\nu}^{*}(T+)\right] \leq \ln \left[M_{\nu}(0-) / M_{\nu}(T+)\right]
$$

Each $M_{\nu}^{*}$ is of one of the types enumerated in Theorem 5.4; hence through possible extraction of subsequences we can suppose all sequences on $\nu$ so chosen that each $M_{\nu}^{*}$, $\nu=1,2, \cdots$, is of the same type and such that $M_{\nu}^{*}$ converges to a limit $M^{*}$ on $[-1$, $T+1$, which is necessarily a step function with at most the same number of steps as $M_{v}^{*}$. Function $\theta_{v}^{*}$ can be taken as constant or linear according as $M^{*}$ has one or two steps. We can suppose $\theta_{\nu}^{*}$ convergent to a limit $\theta^{*}$, again by restriction to suitable subsequences. Terminal position $x_{\nu}^{*}(T), y_{v}^{*}(T)$ and velocity $u_{v}^{*}(T+), v_{\nu}^{*}(T+)$ determined by $M_{v}^{*}, \theta_{v}^{*}$, and $(2.1)$ converge to values (2.2) as observed above.

Passage to the limit in (6.3) shows that

$$
\ln \left[M^{*}(0-) / M^{*}(T+)\right] \leq \ln [M(0-) / M(T+)]
$$

hence that the particular admissible quadruple $\left(M^{*}, \theta^{*}, x^{*}, y^{*}\right)$ is at least as good as $(M, \theta, x, y)$.

Accordingly the minimum problem stated in Sec. 2 has as a solution any solution of the auxiliary minimum problem.

To solve an example refer to the possibilities of Theorem 5.4. Since at most four quantities are subject to four side conditions (3.2) multipliers are not applicable at this stage. One or more solutions to the example are provided by (3.2) with $n=2$.

7. Examples.

$\begin{array}{lllclc}(u, v) & (U, V) & (a, b) & T & \text { type } & c\left(\operatorname{Min} \Sigma \ln r_{i}\right) \\ (0,1) & (0,-2) & (1,0) & 1 & \text { (i) } & 2^{1 / 2}+5^{1 / 2} \\ (1,0) & (2,0) & (2,0) & 1 & \text { (ii) } & 1 \\ (1,0) & (2,0) & (2,0) & 2 & \text { (iii) } & 1 \\ (1,1) & (2,-2) & (2,0) & 2 / 3 & \text { (iv) } & 10^{1 / 2} \\ (1,1) & (4,-2) & (2,0) & 1 & \text { (i) or (iv) } & 3(2)^{1 / 2}\end{array}$


8. Variable transit time. The minimum of $M(0-) / M(T+)$ denoted by $\lambda$ is a function of transit time $T$. If the problem is altered by allowing $T$ to vary over the non-negaitve reals the solution when it exists is available by the present paper plus a minimization with respect to $T$. There are cases in which no minimum exists, e.g. $(u, v)=(0,0),(U, V)=(-1,0),(a, b)=(1,0)$. For each fixed $T$ we have a Type 1 solution. Inf $\lambda(T)$ is approached as $T \rightarrow \infty$ but is never attained.

9. Concluding remarks. The reviewer has called attention to a forthcoming paper by E. W. Graham in the Journal of the Aero/Space Sciences which may overlap the present results and which includes uniform fields. That the uniform field case is mathematically equivalent to the free space problem can be seen as follows. In the first two equations (2.1) add respective constant multiples $2 A T, 2 B T$ of $t$; hence in the last equations (2.1) add $A T^{2}, B T^{2}$. In the respective equations (3.2) we must then replace $a, b$ by $a\left(1-T^{2}\right), b\left(1-T^{2}\right)$ and replace $U, V$ by $U-2 A T$ and $V-2 B T$. Subject only to these substitutions all present results apply.

Among questions for which only partial theories appear to exist are the following.

If terms representing a general gravitational field are included in (2.1) as in [4], for what non-uniform fields is there an analog of Theorem 5.4, eliminating the need for maneuvers with more than two null-thrust arcs like that of $[6$, p. 175, Fig. 6]?

If our problem is modified into Type 2 by adjoining a Lipschitz condition on $M$, is an optimal maneuver realizable with at most two periods of maximal thrust?

With aerodynamic forces also present we have the possibility of time intervals on which $M$ decreases continuously in addition to possible coasting periods together with impulsive burnings or periods of maximal thrust according as the problem is of Type 1 or 2. An extension of Theorem 5.4 coupled with proof of the existence of a best program should be of interest.

\section{BIBLIOGRAPHY}

1. G. A. Bliss, Lectures on the calculus of variations, University of Chicago Press, 1946

2. O. Bolza, Vorlesungen चber Variationsrechnung, Teubner, Leipzig und Berlin, 1909

3. L. M. Graves, The theory of functions of real variables, McGraw-Hill, New York, 1946

4. D. F. Lawden, Minimal rocket trajectories, J. of American Rocket Soc. 23, 360-367 (1953)

5. D. F. Lawden, Dynamics of interplanetary flight, Aeronaut. Quart. 6, 165-180 (1955)

6. D. F. Lawden, Mathematical problems of astronautics, Math. Gaz., 41, 168-179 (1957)

7. A. Miele and C. R. Cavoti, Generalized variational approach to the optimum thrust programming for the vertical fight of a rocket, part II, Z. Flugwissenschaften 6, 102-109 (1958)

8. A. Miele, Flight mechanics and variational problems of a linear type, J. Aero/Space Sci. 25, 581-590 (1958)

9. A. Miele, Mathematical theory of the optimum trajectories of a rocket, Report A-58-10, AFOSR-TR58-174, Purdue University, School of Aeronautical Engineering, Lafayette, Ind., Nov. 1958

10. F. A. Valentine, The problem of Lagrange with differential inequalities as side conditions. in Contributions to the calculus of variations, 1939-87, University of Chicago Press, 1937 